\title{
Variability of the effective strain rate in SHTB tests and related effects on the dynamic stress amplification
}

\author{
Giuseppe Mirone* ${ }^{*}$ Raffaele Barbagallo \\ University of Catania, Department of Civil Engineering and Architecture, 95125 Catania, Italy
}

\begin{abstract}
Although the standard procedure for SHTB testing is based on the hypothesis that the strain rate is nominally constant during each test, it is known by many experimental evidences that the effective strain rate histories cannot be really constant, and this may cause the function expressing the dynamic amplification of the stress to substantially differ from that inferred under the hypothesis of strain rate constancy. This aspect is stressed out in this work by experiments and by numerical analyses, relating the variability of the strain rate to the finite rise time necessary in Hopkinson bars for reaching the plateau of the loading wave, and to the abrupt large increase in the effective strain rate naturally induced by the necking onset. Also the interplay between the achievement of the strain rate plateau and the achievement of the necking initiation strain is analysed, for assessing if and eventually how the same strain rate plateau achieved before or after the necking onset can affect the resulting dynamic amplification of the stress $17 \mathrm{~mm}$ from the left and right page margins and justified. .
\end{abstract}

\section{Introduction}

The effect of the strain rate on the elastoplastic response of metals consists of a dynamic amplification of the equivalent stress, usually modelled by monotonically increasing functions of the strain rate saturating at strain rate values within the range of those achieved in Split Hopkinson Tensile Bar (SHTB) tests, as in the formulations by Johnson-Cook, Cowper-Symonds and Zerilli-Armstrong. The necking under dynamic loading conditions is still an open issue attracting the interest of various researchers. Rusinek et al. related the critical impact velocity to the energy storage capability of specimens and to the position where multiple necking may originate along the specimen axis, Besnard et al. implemented stereo correlation techniques for in-depth necking measurements, while Sato et al. adopted digital image correlation to the same subject for getting detailed distributions of necking-affected strains and fracture surfaces. Osovski et al (2013) examined the effects of sample size and boundary conditions on the necking inception and development in dynamically stretched steel specimens concluding that the local ductility of the material is geometry independent, i.e. it is also insensible to notches. An interaction between the necking, the effective true strain rate and the dynamic amplification was already identified in previous works (Mirone, 2013; Mirone et al., 2017) where it was also found that the necking onset freezes the sensitivity of the stress-strain curve to the strain rate.
In this work, the necking onset and the post necking evolution are firstly investigated under different static and dynamic straining histories.

Then, camera-assisted static/temperature/dynamic experiments by Split Hopkinson tensile bars (SHTB) with AISI304 steel are discussed for investigating how the dynamic amplification evolves beyond the necking.

\section{Stress-strain definitions and general hardening models}

Different arbitrary materials are modelled according to the general model of dynamic hardening in eq. (1).

$$
\sigma_{\mathrm{Eq}}\left(\varepsilon_{\mathrm{Tru}}, \varepsilon_{\mathrm{True}}, \mathrm{T}\right)=\sigma_{\mathrm{Eq}-\mathrm{s}}\left(\varepsilon_{\mathrm{True}}\right) \cdot \mathbb{R}\left(\varepsilon_{\mathrm{True}}\right) \cdot S(\mathrm{~T})
$$

where the static hardening, the dynamic amplification and the thermal softening $\mathrm{S}$ are uncoupled each other. In this first series of analyses the thermal softening is neglected by considering that $S(T)=1$, while two static hardenings and two dynamic amplifications are combined each other leading to four different materials.

The two selected functions for static hardening, EN and LN, respectively exhibit late necking ( $\varepsilon_{\mathbb{N}} \approx 0.31$ ) and early necking $\left(z_{N} \leqslant 0.025\right)$; both functions are plotted in the left side of Fig. 1 and are expressed by eqs. (2) and (3). 


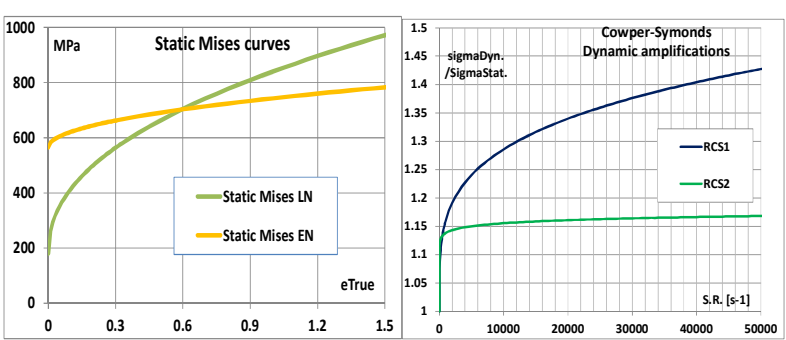

Fig. 1. Static Mises curves and Dynamic amplifications.

EN static curve:

$\sigma_{\mathrm{Bq}_{5}}\left(\varepsilon_{\text {True }}\right)=565+178 \cdot \varepsilon_{\text {True }}{ }^{0.5} z_{s} z_{\mathrm{N}} \& 0.025:(2)$

LN static curve:

$\sigma_{\text {Eq_s }}\left(\varepsilon_{\text {True }}\right)=180+660 \cdot \varepsilon_{\text {True }}{ }^{0.45} ; \varepsilon_{N} \approx 0.31 ;(3)$

Two arbitrary dynamic amplifications according to the Cowper-Symonds model, CS1 and CS2, are plotted on the right in Fig. 1 and are expressed by eqs. (4) and (5),

CS1 monotonically increases up to 50 '000 s-1, while CS2 saturates and just evolves between $13 \%$ at $\boldsymbol{s}_{\text {True }}=$ $500 \mathrm{~s}-1$ and $18 \%$ at $\boldsymbol{\varepsilon}_{\text {True }}=50 \prime 000 \mathrm{~s}-1$.

CS1 dynamic amplification:

$$
\mathbb{R}\left(\hat{e}_{\text {True }}\right)=1+\left(\frac{t}{1,510^{5}}\right)^{\text {ans }}
$$

CS2 dynamic amplification:

$$
R\left(\dot{e}_{\text {True }}\right)=1+\left(\frac{t}{1,5 \cdot 10^{20}}\right)^{0,05}
$$

The four arbitrary materials resulting from the combinations of the two static hardenings with the two dynamic amplifications are then identified as LN-CS1, LN-CS2, EN-CS1 and EN-CS2.

\section{Criteria of instability and necking onset}

The onset of necking and tensile instability is firstly addressed here. In case of static loading, $\sigma_{\mathrm{Eq}}$ only depends on $\varepsilon_{\text {True }}$ and the well-known Considère strain triggers instability and necking:

$$
\sigma_{E q}-\frac{\partial \sigma_{\mathrm{Eq}}}{\partial z_{\mathrm{Trus}}}=0 ; \rightarrow \varepsilon_{\mathrm{M}}
$$

In case of dynamic loading, $\sigma_{E \text { g }}$ depends on both $\varepsilon_{\text {True }}$ and $\dot{\varepsilon}_{\text {True }}$, while, at the same time, $\dot{\varepsilon}_{\text {True }}$ depends on $\varepsilon_{\text {True }}$. Then, the instability condition based on the total derivative yields:

$$
\frac{a p}{\partial z_{\text {Truz }}}=\frac{\partial\left(\sigma_{\mathrm{Eq}} A\right)}{\partial z_{\mathrm{Truz}}}=\mathrm{A} \cdot \frac{\partial\left(\sigma_{\mathrm{Eq}}\right)}{\partial z_{\mathrm{TruE}}}+\sigma_{\mathrm{Eq}} \cdot \frac{a(\mathrm{~A})}{\partial z_{\mathrm{Truz}}}=0
$$

The cross section $A$ directly depends on $\varepsilon_{\text {TruE }}$, then the last derivative in (7) can be expressed in terms of srue:

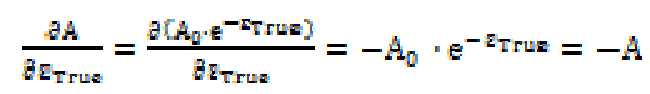

by writing the partial derivatives with respect to $\varepsilon_{\text {True }}$ and $\varepsilon_{\text {True }}$ yields:

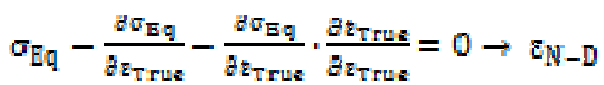

Eq. (9) returns the most general instability strain. From the Hart condition (Hart, 1967) for uniform undamaged cross sections, the shrinking speed of the cross section is maximum at the neck section:

$$
B A / b z=0
$$

Eq. (10) implies that, at the onset of necking, the shrinking speed $A$ is maximum at the neck section. Expressing partial derivatives by the chain rule returns the Hart's condition for necking onset:

$$
\sigma_{E q}-\frac{d \sigma_{\mathrm{Eq}}}{\partial z_{\mathrm{TruE}}}-\frac{d \sigma_{\mathrm{Eq}}}{\partial \tau_{\mathrm{Truz}}} \cdot \hat{\varepsilon}_{\mathrm{True}}=0 \rightarrow \varepsilon_{\mathrm{N}-\mathrm{H}}
$$

Instead, the Ghosh approach (Ghosh, 1977) is based on the hypothesis that the elongation speed $\mathbb{L}$ is constant as the plastic strain flows and instability takes place:

$$
\frac{a L}{a \tau_{\text {Tus }}}=0
$$

Relating the strain to the elongation and making explicit the partial derivatives returns the Ghosh criterion for the necking onset:

$$
\sigma_{\mathrm{Eq}}-\frac{d \sigma_{\mathrm{Eq}}}{\partial z_{\mathrm{Truz}}}+\frac{d \sigma_{\mathrm{Eq}}}{\partial z_{\mathrm{Trus}}} \cdot \vec{z}_{\mathrm{True}}=0 \rightarrow \mathrm{z}_{\mathrm{W}-\mathrm{G}}
$$

The necking initiation and evolution is now investigated by applying the above conditions to different straining histories typical of SHTB tests, simulated by f.e. referring to the four materials functions previously discussed.

\section{Instability criteria and macroscopic necking indicators from F.E.}

The input and output bars of a SHTB system are modelled by Finite Elements (F.E.) with the elastic properties of $\mathrm{Al} 7075$. The tensile specimen has $3 \mathrm{~mm}$ diameter, $7.5 \mathrm{~mm}$ gauge length and round fillets connecting the gauge length to the shoulders and the connection to the bars. Incident waves are assigned as time-dependent pressure functions at the free end of the input bar and are let free of propagating until the specimen is loaded up to the desired amount of strain.

A trapezoidal incident wave of $90 \mathrm{kN}$ with 50 microseconds of rise time is modelled for each analysis.

From each F.E. analysis, the evolving $\varepsilon_{E q}, \varepsilon_{E q}, \sigma_{E q}$ and $\sigma_{z}$ are calculated at the neck centre, together with the whole specimen elongation. Such data are reported in Figures 2 to 5 .

The first plot a) in each figure reports the lengthbased and the diameter-based strain rates, each plotted against the respective elongation-based or diameterbased strain. Both strain rates are almost equal each other before the necking while largely diverge as necking and localization takes place. Then the 
bifurcation of such strain rates is a macroscopic evidence of necking occurrence.

The second plot b) in each figure shows $\sigma_{\mathrm{Eq}}$ and $\sigma_{z}$ plotted against $\varepsilon_{\mathrm{Eq}_{\mathrm{q}}}$. These stresses are identical each other before necking while significantly diverge each other as the necking-induced triaxiality progresses. Then the bifurcation of $\sigma_{\mathrm{Eq}}$ from $\sigma_{2}$ too indicates that the necking is taking place.

The third plot c) in each figure reports the left hand side of eqs. (10) (18) and (19), so that the points where the curves cross the abscissa deliver the instability strains according to the general dynamic criteria, the Gosh criteria and the Hart criteria, respectively.
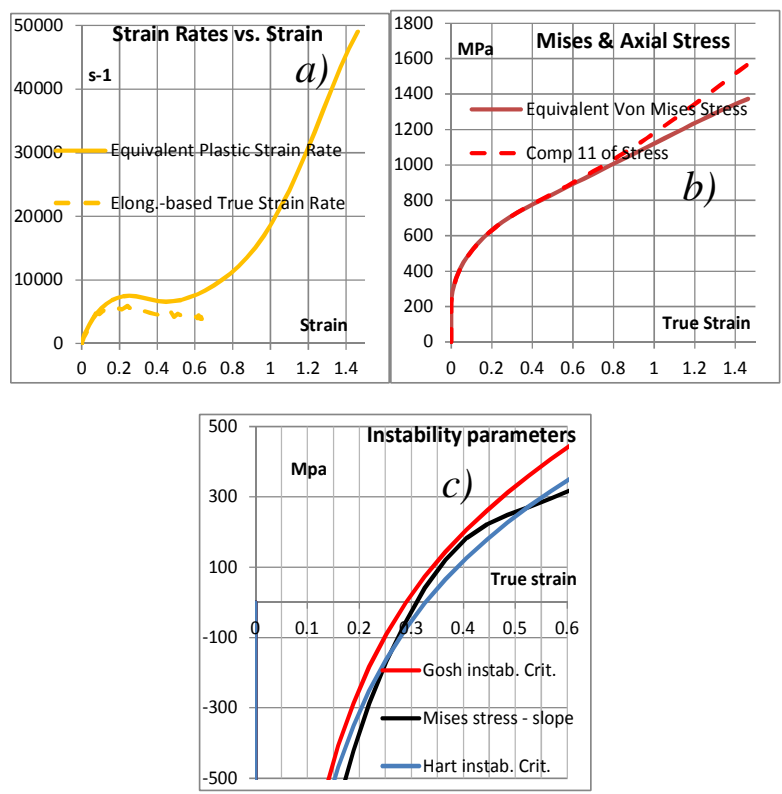

Fig. 2. Variables for necking onset of LN-CS1, wave 90kN@50micros.

Firstly, all the analyses confirm that the effective strain rate increases after the necking onset up to 5-10 times more than the nominal value, as already pointed out in Mirone (2013) anf Mirone et al. (2017). Whatever strain history in SHTB testing can be then controlled by the incident wave only up to the necking onset; beyond such limit, $\dot{\varepsilon}_{\text {Truf }}$ undergoes a spontaneous uncontrollable necking-induced increase up to failure.

Summarising, Figures 2 to 5 show that the macroscopic necking effect may be delayed with respect to the instability; such two aspects are then discussed separately.

While the instability condition can be found by mathematics, the macroscopic necking effect is always gradual and cannot be exactly pinpointed. Thus, a qualitative reference for the beginning of such macroscopic necking effect is $\boldsymbol{a}_{\mathrm{N}-\text { Macro }}$, placed at the visible divergence point of $\sigma_{Z}$ from $\sigma_{\mathrm{Eq}}$.

It is confirmed that the instability conditions of Hart and Ghosh are approximate, then only $\varepsilon_{\mathrm{N}-\mathrm{D}}$ is considered as the effective dynamic instability strain. Fig.6 provides further useful insight of the discussed phenomena, by showing the deformed specimens from the four dynamic analyses above compared to those of two static analyses, respectively with the LN and the EN flow curves alone; all pictures are taken as the maximum local strain at the neck centre is close to 1.0.
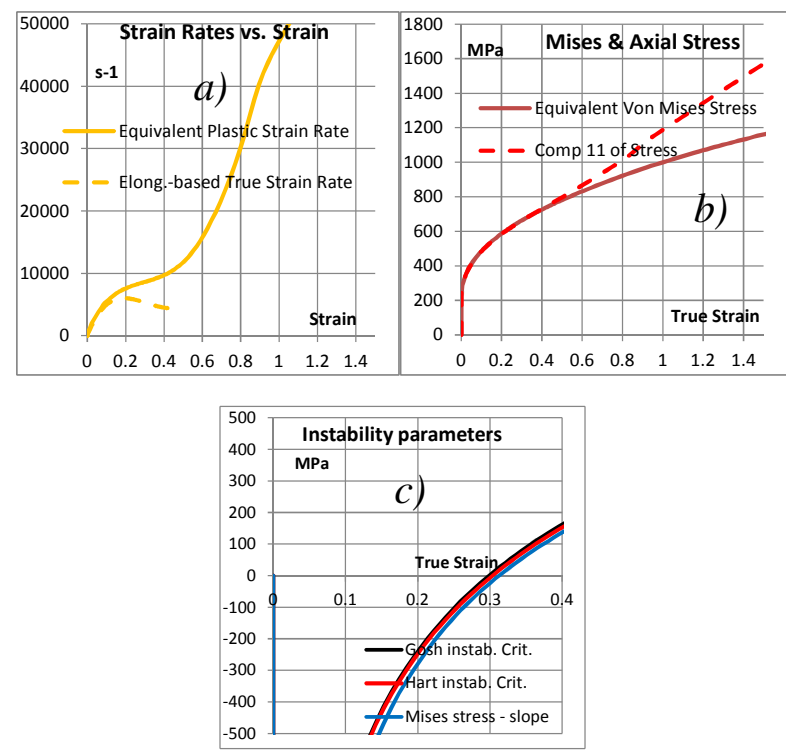

Fig. 3. Variables for necking onset of LN-CS2, wave 90kN@50micros.
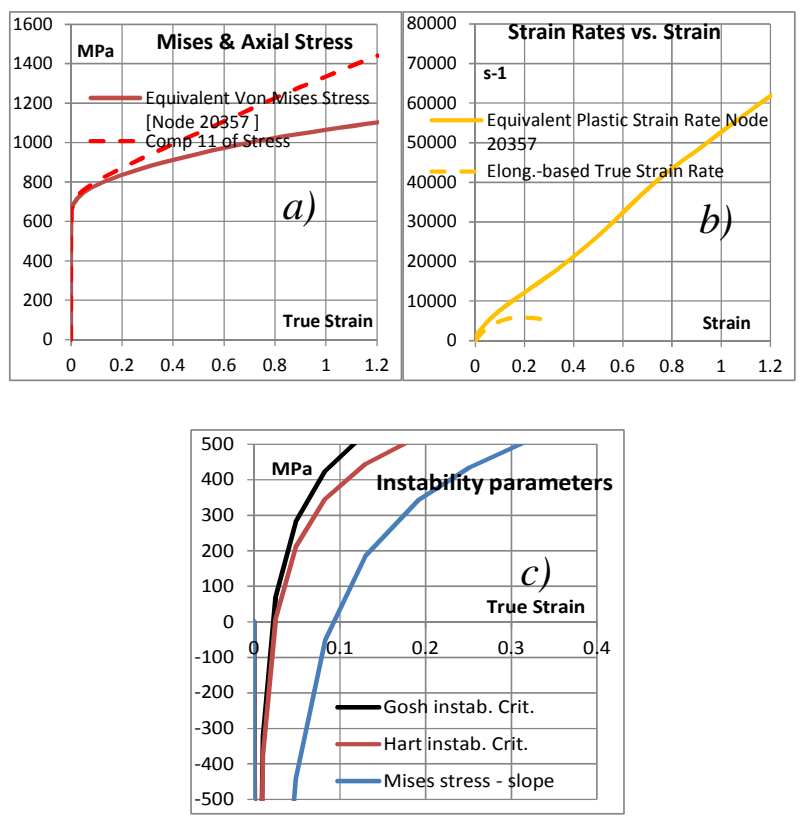

Fig. 4. Variables for necking onset of EN-CS1, wave 90kN@50micros.

The static simulations can represent the limit cases of vanishing dynamic amplifications with $\mathbb{R}\left(\varepsilon_{\text {True }}\right)=1$. Fig.6 further confirm that the higher is the dynamic amplification the less pronounced is the curvature of the necking profile; this, in turn, means lower strain localization and lower stress triaxiality at the same given post-necking strain.

This outcome also agrees with previous results from Mirone et al. (2017) and can be qualitatively related to the phenomenon of super plasticity too. 


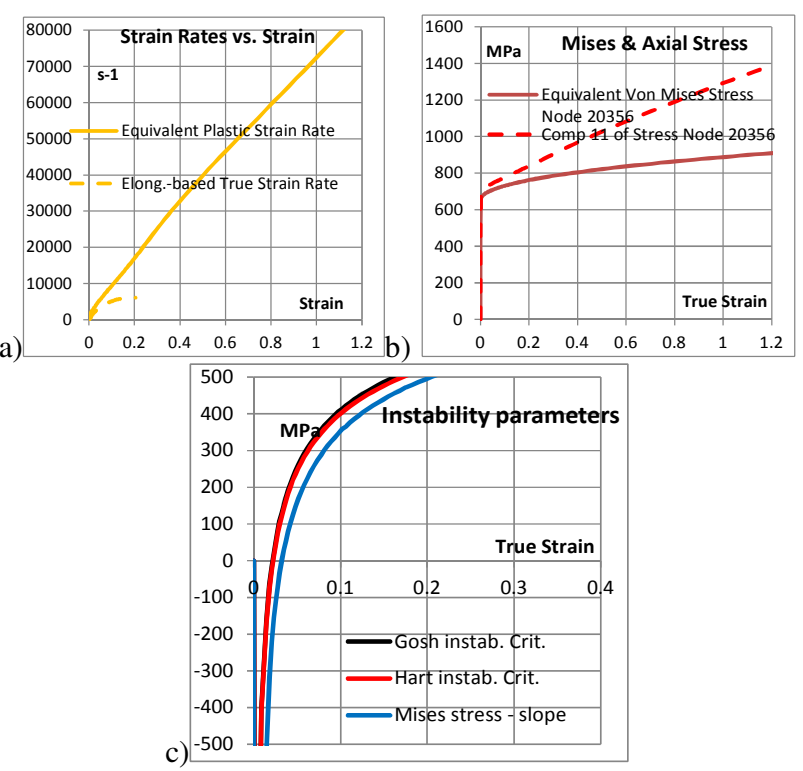

Fig. 5. Variables for necking onset of EN-CS2, wave 90kN@50micros.

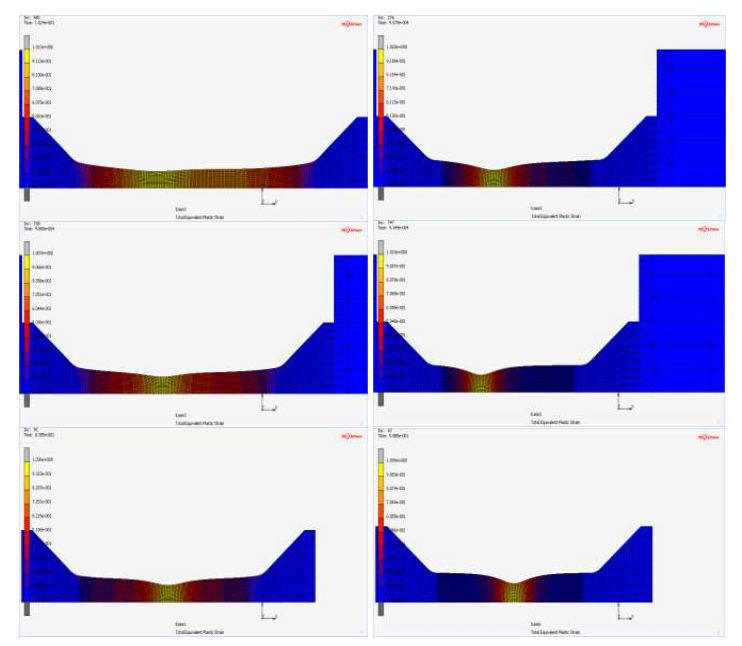

Fig.6. Deformed shapes of LN static curve with different dynamic amplifications - peak strain 1.0.

\section{Static Dynamics and mixed experiments with AISI 304}

Round tensile specimens of AISI 304 are machined with nominal $3 \mathrm{~mm}$ diameter and $9 \mathrm{~mm}$ nominal length of the constant cross section segment. Static tensile tests are carried out by motor driven machines at static rate (0.003 s-1) under different temperatures $\left(20{ }^{\circ} \mathrm{C}, 100{ }^{\circ} \mathrm{C}\right.$ and $150{ }^{\circ} \mathrm{C}$ ).

Dynamic tests are carried out by the direct-tension split Hopkinson tension bar (SHTB) described in Mirone et al. (2017), with Al7075 bars of 16 mm diameter.

Two series of SHTB tests are ran: the "dynamic" D tests are conventionally performed with an incident wave of $17.5 \mathrm{kN}$ (preload $35 \mathrm{kN}$ ) on the machined specimens, while the "static/dynamic" S/D tests are ran, with the same incident wave, on specimens previously statically pre-strained just beyond the necking. From each test, $t, \mathrm{P}$ and $\mathrm{d}$ (time, load and minimum cross section diameter) are determined; speed and conventional camera acquisitions together with testing machine and strain gauge recordings provided the necessary information.

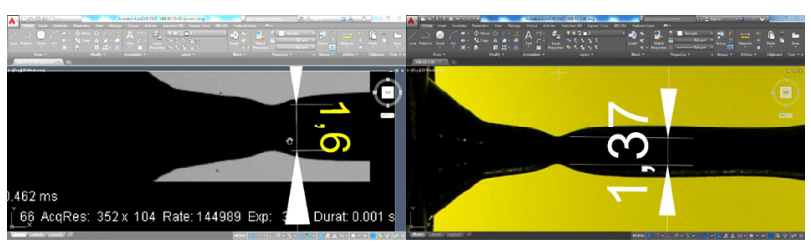

Fig. 7. Image analysis of cameras frames.

The static tests at different temperatures just delivered discrete true stress-true strain points according to eqs. (20) and (21):

$$
\begin{aligned}
& \sigma_{\text {True }}=\frac{F}{\pi / 4 d^{2}} \\
& \varepsilon_{\text {True }}=2 \cdot \operatorname{Ln}\left(\frac{d a}{d}\right)
\end{aligned}
$$

The dynamic tests delivered the same data above, plus the time derivative of the best fit function $\varepsilon_{\text {True }}(t)$ delivering the strain rate:

$$
\varepsilon_{\text {True }}=\frac{g_{\mathrm{r}_{\text {Trus }}}[\mathrm{t}}{\partial \mathrm{t}}
$$

The true stress-true strain data from static and dynamic tests are shown in Fig. 8 together with the corresponding best fit curves.

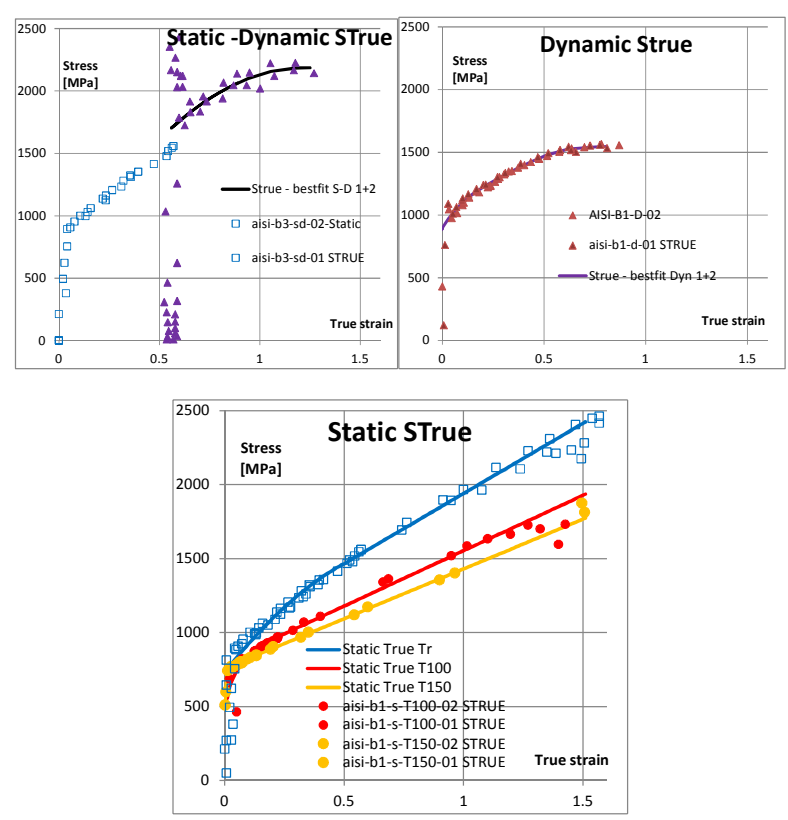

Fig. 8. Exp. true stress-true strain data with best fit curves.

The static curves are firstly transformed into Mises curves by the MLR correction, so that the ratio $\sigma_{\mathrm{Eq}}(\mathrm{T}) / \sigma_{\mathrm{Eq}}\left(\mathrm{T}_{\mathrm{Room}}\right)$ can be calculated at each strain level. The left side of Fig. 9 reports the softening at different strains from the tests at $100{ }^{\circ} \mathrm{C}$ and $150{ }^{\circ} \mathrm{C}$; apart from a transitory phase between yielding and necking, the softening ratio a given temperature is nicely constant, so that these constant values are associated to the corresponding temperatures; a Johnson-Cook-like softening function with exponent 0.5 is then found to reasonably approximate the data at 100 and $150{ }^{\circ} \mathrm{C}$ 
together with the trivial points corresponding to the softening at room temperature and at melting temperature $\left(1450{ }^{\circ} \mathrm{C}\right)$.

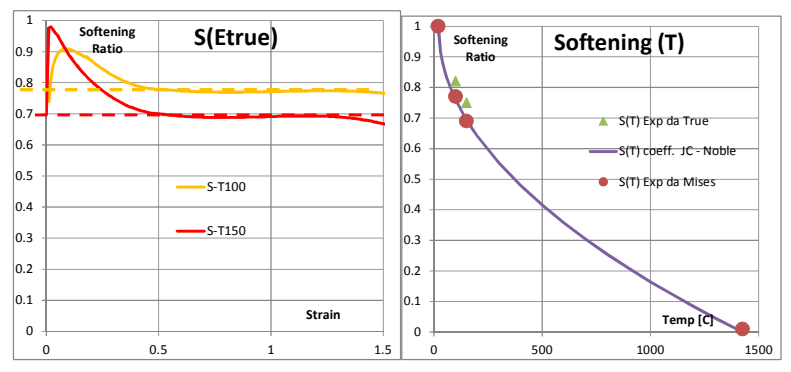

Fig. 9. Softening function.

The softening $\mathbf{S ( T )}$ so identified form static tests, is then supposed to still apply to the temperature increase due to adiabatic plastic heating of dynamic tests.

Then, the true curves from tests $\mathrm{D}$ and S/D are backcorrected by $\mathbf{S ( T )}$ and by the necking correction MLR, so that the Mises stress only affected by the dynamic amplification is obtained.

The starting true curves and the final Mises curves are plotted together in Fig. 10.

For completing the insight on these experiments, the strain rate - vs. - strain relationship is obtained according to eq. (23); the starting data and the final evolving strain rates are reported in Fig. 11.

Finally, by joining the information from Fig. 10 and Fig. 11 the long-expected dynamic amplification can be obtained; in fact, at each strain level, the ratio of dynamic to static Mises stresses from Fig. 10 can be associated to the current strain rate from Fig. 11; the outcome of such process is shown in Fig. 12.

The analysis of such data allows deriving some useful considerations about the evolution of the dynamic stress amplification in the post necking range.

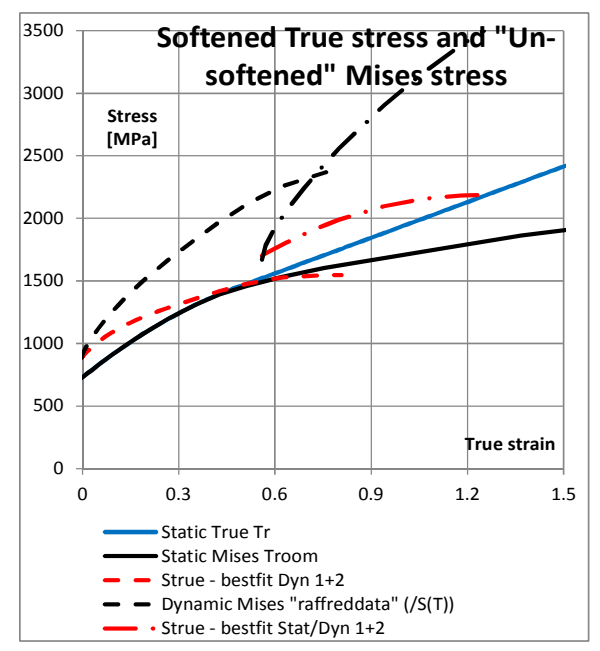

Fig. 10. Exp. true curves and "back-corrected" Mises curves.

Fig. 12 shows that the dynamic amplification $\mathbf{R}\left(\varepsilon_{\text {Tna }}\right)$ derived from the D tests starts at about 1.2 at first yield, then quickly increases up to about 1.4 at 1000 $\mathrm{s}^{-1}$ and then abruptly breaks, becoming nearly constant until failure. As visible on the right side of Fig. 11, the strain rate of $1000 \mathrm{~s}^{-1}$ at which the above breaking occurs is the plateau strain rate of tests D, usually addressed as the nominal strain rate, extending from the end of the rise time of the incident wave until the necking takes place. This agrees with the findings from Mirone et al. (2017), where similar results were inferred via other considerations for very early-necking steel, leading to the concept of "freezing" of the dynamic amplification caused by the necking onset.

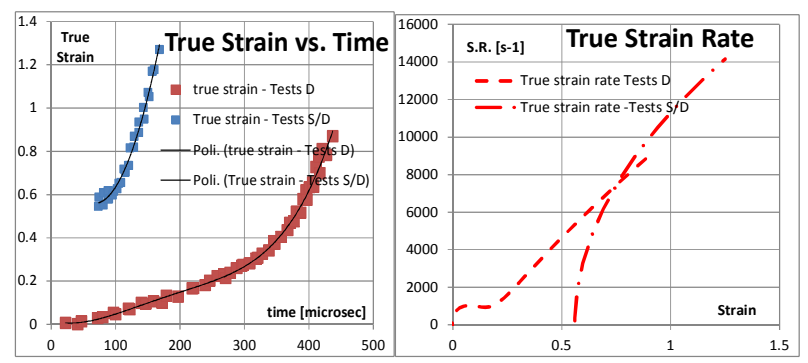

Fig. 11. True strain and True strain rate.

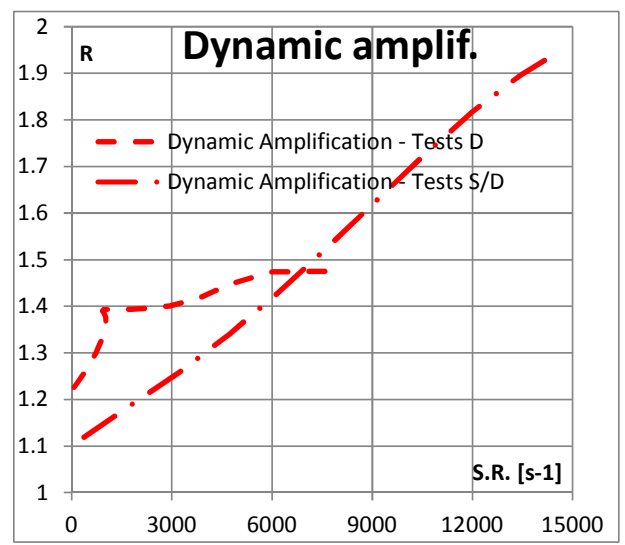

Fig. 12. Dynamic amplification.

So far, without further support of any numerical verification, the breaking of $\mathbb{R}\left(\hat{s}_{\text {Trus }}\right)$ might be attributed to a saturating attitude of the dynamic amplification, which is commonly assumed to be a material property in standard models of dynamic hardening, independently of the strain history and of the stress state.

Instead, the dynamic amplification derived from the S/D test shows that the necking-induced changes of the stress state generate significant changes in the dynamic amplification.

In fact, the main difference between the curves in Fig. 12 is that $\mathbb{R}\left(\dot{\varepsilon}_{\mathrm{True}}\right)$ from $\mathrm{S} / \mathrm{D}$ tests monotonically increases without freezing at any strain rate, while $\mathbb{R}\left(\varepsilon_{\text {True }}\right)$ from the S/D tests clearly does.

Although no certainty is currently available for justifying such behaviour, the only reasonable explanation found so far is that in D tests the load instability condition occurs while plastic strain flows, while in dynamic S/D tests of pre-necked specimens the instability occurs at the threshold between elastic and plastic range. According to this hypothesis, the freezing might only show up if the necking occurs during the plastic flow at high strain rates.

The alternative to such hypothesis should be that a remarkable coupling exists between the effects of $\varepsilon_{\text {True }}$ 
and $\dot{\varepsilon}_{\text {Truf }}$, which contradicts all known models of dynamics hardening.

Two more points are worth noting, which support the overall validity of the proposed framework.

The first point is that the temperature, at the beginning of $\mathrm{D}$ and $\mathrm{S} / \mathrm{D}$ experiments, is still close to 20C; then both tests at their beginning are not affected by any temperature-softening issue, thus the corresponding ratios of experimental stresses deliver the real effective dynamic amplifications, without any uncertainty exceeding those of usual experimental accuracy.

With reference to Fig. 11 and Fig. 12, this means that the stress of just-machined specimens undergoing $\varepsilon_{\text {True }} \$ 1000$ s-1 before necking $\left(\varepsilon_{\text {True }} \propto 0.1\right)$ is amplified more than $40 \%$ with respect to the static case; instead, the stress of statically pre-necked specimens undergoing the same $\varepsilon_{\text {True }} \approx 1000 \mathrm{~s}-1$ (at $\varepsilon_{\text {True }} \approx 0.55$ ) is just amplified of a mere $15 \%$ with respect to the static case.

It is evident that either the necking affects the dynamic amplification and the freezing effect show up whenever the instability occurs during plastic flow, or a huge coupling of $\boldsymbol{\varepsilon}_{\text {True }}$ and $\boldsymbol{\varepsilon}_{\text {True }}$ exists.

The second observation regards the intersection of the relevant curves in Fig. 10, Fig. 11 and Fig. 12.

When the strain is close to 0.75 , both $\mathrm{D}$ and S/D tests exhibit the same strain rate of about $7600 \mathrm{~s}-1$ (right side of Fig. 11) and both tests are in the post-necking condition, then no reason should exist for them delivering different Mises stresses and different dynamic amplifications: in fact, the Mises curves from D ans S/D tests intersect each other at a strain of 0.75 (black curves in Fig. 10) and, as a consequence, the $\mathbf{R}\left(\varepsilon_{\text {Tru }}\right)$ curves from $\mathrm{D}$ and from $\mathrm{S} / \mathrm{D}$ tests in Fig. 12 intersect each other at a strain rate of about $7600 \mathrm{~s}-1$. While the intersection of $\mathbb{R}\left(\varepsilon_{\text {True }}\right)$ curves is trivial, that of the Mises curves is a purely experimental outcome which nicely supports the overall explanation proposed here.

\section{Conclusions}

The onset of instability and necking in dynamic tensile tests is firstly analysed with reference to arbitrary materials showing extreme features in terms of early-late static necking and saturating-monotonically increasing dynamic amplification.

While being quite accurate in many cases, the Gosh and the Hart conditions for instability have been found to be just moderately accurate in case of materials exhibiting early necking and steep dynamic amplifications of the stress. Furthermore, the macroscopic effect of the necking, consisting of the curvature of the profile with consequent strain localization and development of stress triaxiality, is found to be more or less delayed with respect to the occurrence of instability condition.

A series of experiments has been carried out for assessing the static and the dynamic Mises curves together with the thermal softening. Further special tests of statically pre-necked specimens also returned postnecking true curves and dynamic amplifications.

The crossing of such experimental results seems to confirm that when the necking occurs while plastic straining is in progress, the dynamic amplification is frozen from the necking up to failure. When instead, the necking occurs within the first yield, as for the prenecked specimens, no freezing occurs and the dynamic amplification evolves monotonically up to failure.

Further experiments are in progress in order to extend the above results and to gain deeper insight in the problems of dynamic stress-strain characterization.

\section{References}

1. Besnard G., Hild F., Lagrange J. M. , Martinuzzi P., Roux S., Analysis of necking in high speed experiments by stereocorrelation, International Journal of Impact Engineering. 49: 1353-1367 (2012)

2. Bridgman, P. W., Studies in large plastic flow and fracture (Vol. 177). New York: McGraw-Hill, (1952)

3. Lin, E.I.H., Plastic instability criteria for necking of bars and ballooning of tubes, Int. conf. on structural mechanics in reactor technology, San Francisco, (1977)

4. Hart EW., Theory of the tensile test, Acta Metall; 15:351-5. (1967)

5. Guan Z., Quantitative analysis on the onset of necking in rate-dependent tension, Materials and Design 56 209-218 (2014)

6. Ghosh, A. K. Tensile instability and necking in materials with strain hardening and strain-rate hardening. Acta Metallurgica, 25 (12), 1413-1424 (1977)

7. Mirone, G., \& Barbagallo, R. Congelamento dello Strain Rate Effect In Prove Dinamiche su Hopkinson Bar. Proceedinigs of the $46^{\text {th }}$ AIAS National Conference, Pisa (2017)

8. Mirone G., Corallo D., Barbagallo R., Experimental issues in tensile Hopkinson bar testing and a model of dynamic hardening. Int. J. of Impact Engineering, 103, 180-194 (2017)

9. Mirone, G. A new model for the elastoplastic characterization and the stress-strain determination on the necking section of a tensile specimen. International Journal of Solids and Structures, 41(13), 3545-3564 (2004)

10. Mirone G., The dynamic effect of necking in Hopkinson bar tension tests, Mech. Mater. 58 8496 (2013)

11. Osovski, S., Rittel, D., Rodríguez-Martínez, J. A., \& Zaera, R., Dynamic tensile necking: influence of specimen geometry and boundary conditions. Mechanics of materials, 62, 1-13 (2013)

12. Rusinek A. ,Zaera R., Klepaczko J. R., Cheriguene R., Analysis of inertia and scale effects on dynamic neck formation during tension of sheet steel, Acta Mater.; 53:5387-400 (2005) 